
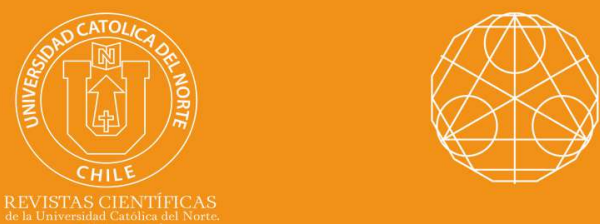

\title{
Some hyperstability results of a $p$-radical functional equation related to Drygas mappings in non-Archimedean Banach spaces
}

Mustapha Esseghyr Hryrou ${ }^{1}$

Ahmed Nuino ${ }^{2}$

Samir Kabbaj $^{3}$ (1) orcid.org/0000-0001-9717-9366

Ibn Tofaïl University, Dept. of Mathematics, Faculty of Sciences, Kenitra, Morocco.

1曰hryrou.mustapha@hotmail.com;2区ahmed.nuino2015@gmail.com;

3曰samkabbaj@yahoo.fr

Received: November 2019 | Accepted: December 2020

Abstract:

The aim of this paper is to introduce and solve the following $p$ radical functional equation related to Drygas mappings

$$
f\left(\sqrt[p]{x^{p}+y^{p}}\right)+f\left(\sqrt[p]{x^{p}-y^{p}}\right)=2 f(x)+f(y)+f(-y), \quad x, y \in \mathbf{R},
$$

where $f$ is a mapping from $\mathbf{R}$ into a vector space $X$ and $p \geq 3$ is an odd natural number. Using an analogue version of Brzdȩk's fixed point theorem [12], we establish some hyperstability results for the considered equation in non-Archimedean Banach spaces. Also, we give some hyperstability results for the inhomogeneous p-radical functional equation related to Drygas mappings

$$
f\left(\sqrt[p]{x^{p}+y^{p}}\right)+f\left(\sqrt[p]{x^{p}-y^{p}}\right)=2 f(x)+f(y)+f(-y)+G(x, y) .
$$

Keywords: Hyperstability; non-Archimedean Banach spaces; Radical functional equations; Drygas functional equations.

MSC (2020): 39B82, 39B62, 47H14, 47J20, 47H10.

Cite this article as (IEEE citation style):

M. E. Hryrou, A. Nuino, and S. Kabbaj, "Some hyperstability results of a p-radical functional equation related to Drygas mappings in non-Archimedean Banach spaces", Proyecciones (Antofagasta, On line), vol. 40, no. 1, pp. 153-174, 2021, doi: 10.22199/issn.0717-6279-2021-01-0010

Article copyright: (C) 2021 Mustapha Esseghyr Hryrou, Ahmed Nuino, and Samir Kabbaj. This is an open access article distributed under the terms of the Creative Commons License, which permits unrestricted use and distribution provided the original author and source are credited. 


\section{Introduction}

A classical question in the theory of functional equation is the following: "When is it true that a function which approximately satisfies a functional equation must be close to an exact solution of this equation."

If the answer is affirmative, then we say that equation is stable. In 1940, S. M. Ulam [37]) asked the following question concerning the stability of group homomorphisms

Let $\left(G_{1}, *_{1}\right)$ be a group and let $\left(G_{2}, *_{2}\right)$ be a metric group with a metric $d(.,$.$) . Given \varepsilon>0$, does there exists a $\delta>0$ such that if a mapping $h: G_{1} \rightarrow G_{2}$ satisfies the inequality

$$
d\left(h\left(x *_{1} y\right), h(x) *_{2} h(y)\right)<\delta
$$

for all $x, y \in G_{1}$, then there exists a homomorphism $H: G_{1} \rightarrow G_{2}$ with

$$
d(h(x), H(x))<\varepsilon
$$

for all $x \in G_{1}$ ?

This question seems to be the starting point of studying the stability of functional equations. Since then, this question has attracted the attention of many researchers. The first partial answer was raised by D. H. Hyers [24] in 1941 under the assumption that $G_{1}$ and $G_{2}$ are Banach spaces for the the additive functional equation as follows:

Theorem 1.1.[24] Let $E_{1}$ and $E_{2}$ be two Banach spaces and $f: E_{1} \rightarrow E_{2}$ be a function such that

$$
\|f(x+y)-f(x)-f(y)\| \leq \delta
$$

for some $\delta>0$ and for all $x, y \in E_{1}$. Then the limit

$$
A(x)=\lim _{n \rightarrow \infty} 2^{-n} f\left(2^{n} x\right)
$$

exists for each $x \in E_{1}$, and $A: E_{1} \rightarrow E_{2}$ is the unique additive function such that

$$
\|f(x)-A(x)\| \leq \delta
$$

for all $x \in E_{1}$. Moreover, if $f(t x)$ is continuous in $t$ for each fixed $x \in E_{1}$, then the function $A$ is linear. 
Later, T. Aoki [8] and D. G. Bourgin [9] considered the problem of stability with unbounded Cauchy differences. In 1978, Th. M. Rassias [31] attempted to weaken the condition for the bound of the norm of Cauchy difference $\|f(x+y)-f(x)-f(y)\|$ and proved a generalization of Theorem1.1 by using a direct method (cf. Theorem 1.2):

Theorem 1.2.[31] Let $E_{1}$ and $E_{2}$ be two Banach spaces. If $f: E_{1} \rightarrow E_{2}$ satisfies the inequality

$$
\|f(x+y)-f(x)-f(y)\| \leq \theta\left(\|x\|^{p}+\|y\|^{p}\right)
$$

for some $\theta \geq 0$, for some $p \in \mathbf{R}$ with $0 \leq p<1$, and for all $x, y \in E_{1}$, then there exists a unique additive function $A: E_{1} \rightarrow E_{2}$ such that

$$
\|f(x)-A(x)\| \leq \frac{2 \theta}{2-2^{p}}\|x\|^{p}
$$

for each $x \in E_{1}$. If, in addition, $f(t x)$ is continuous in $t$ for each fixed $x \in E_{1}$, then the function $A$ is linear.

After then, Th. M. Rassias [32],[33] motivated Theorem 1.2 as follows:

Theorem 1.3. [32],[33] Let $E_{1}$ be a normed space, $E_{2}$ be a Banach space, and $f: E_{1} \rightarrow E_{2}$ be a function. If $f$ satisfies the inequality

$$
\|f(x+y)-f(x)-f(y)\| \leq \theta\left(\|x\|^{p}+\|y\|^{p}\right)
$$

for some $\theta \geq 0$, for some $p \in \mathbf{R}$ with $p=1$, and for all $x, y \in E_{1}-\left\{0_{E_{1}}\right\}$, then there exists a unique additive function $A: E_{1} \rightarrow E_{2}$ such that

$$
\|f(x)-A(x)\| \leq \frac{2 \theta}{\left|2-2^{p}\right|}\|x\|^{p}
$$

for each $x \in E_{1}-\left\{0_{E_{1}}\right\}$.

Note that Theorem 1.3 reduces to Theorem 1.1 when $p=0$. For $p=1$, the analogous result is not valid. Also, J. Brzdęk [11] showed the estimation(1.2) is optimal for $p \geq 0$ in the general case.

In 1994, P. Găvruţa[23] provided a further generalization of Rassias theorem in which he replaced the bound $\theta\left(\|x\|^{p}+\|y\|^{p}\right)$ in (1.1) by a general control function $\varphi(x, y)$ for the existence of a unique linear mapping. 
Recently, J. Brzdȩk [15] showed that Theorem 1.3 can be significantly improved. Namely, in the case $p<0$, each $f: E_{1} \rightarrow E_{2}$ satisfying (1.1) must actually be additive. This result is called the hyperstability of Cauchy functional equation. However, the term of hyperstability was introduced for the first time probably in [28] and it was developed with the fixed point theorem of Brzdȩk in [12]. There after, the hyperstability of a several functional equations have been studied by many authors (see, for example, $[5,7,2,15,28]$ ).

In 2013, Brzdȩk [14] improved, extended and complemented several earlier classical stability results concerning the additive Cauchy equation (in particular Theorem 1.3). Over the last few years, many mathematicians have investigated various generalizations, extensions and applications of the Hyers-Ulam stability of a number of functional equations (see, for instance, [16], [17] and references therein).

Characterizing quasi-inner product spaces, H. Drygas considers in [19] the functional equation

$$
f(x)+f(y)=f(x-y)+\left\{f\left(\frac{x+y}{2}\right)-f\left(\frac{x-y}{2}\right)\right\}, \quad x, y \in \mathbf{R}
$$

which can be reduced to the following equation [34, Remark 9.2, p. 131]

$$
f(x+y)+f(x-y)=2 f(x)+f(y)+f(-y), \quad x, y \in \mathbf{R} .
$$

This equation is known in the literature as Drygas equation and is a generalization of the quadratic functional equation $\mathrm{f}(\mathrm{x}+\mathrm{y})+\mathrm{f}(\mathrm{x}-\mathrm{y})=2 \mathrm{f}(\mathrm{x})+2 \mathrm{f}(\mathrm{y})$, $\mathrm{x}, \mathrm{y} \in \mathbf{R}$. The general solution of Drygas equation was given by B. R. Ebanks, P. L. Kannappan and P. K. Sahoo in[20]. It has the form $\mathrm{f}(\mathrm{x})=\mathrm{A}(\mathrm{x})+\mathrm{Q}(\mathrm{x})$, $\mathrm{x} \in \mathbf{R}$, where $A: \mathbf{R} \rightarrow \mathbf{R}$ is an additive function and $Q: \mathbf{R} \rightarrow \mathbf{R}$ is a quadratic function, see also [25]. A set-valued version of Drygas equa- tion was considered by W. Smajdor in [36].

Recently, the hyperstability of the Drygas functional equation has been studied in [29], [35] and [6].

During the $16^{\text {th }}$ International Conference on Functional Equations and Inequalities (Bȩdlewo, Poland, May 17-23, 2015), W. Sintunavarat presented a talk concerning the Ulam type stability (for information and further references concerning this notion see, e.g., [10]) of the so-called radical functional equation

$$
f\left(\sqrt{x^{2}+y^{2}}\right)=f(x)+f(y)
$$


in the class of real functions. A question of J. Schwaiger about the general solution of the equation was answered a bit later by the author of this paper (see [30], p. 196). In this regard, many papers concerning the solutions and stability of radical functional equations have been established (the readercan refer, for example, to [1, 2, 4, 21, 22, 26, 27]).

Let us recall (see, for instance, [26]) some basic definitions and factsconcerning non-Archimedean normed spaces.

Definition 1.4. By a non-Archimedean field we mean a field $\mathbf{K}$ equipped with a function (valuation) $|\cdot|: \mathbf{K} \rightarrow[0, \infty)$ such that for all $r, s \in \mathbf{K}$, the following conditions hold:

1. $|r|=0$ if and only if $r=0$,

2. $|r s|=|r||s|$,

3. $|r+s| \leq \max \{|r|,|s|\}$.

The pair $(\mathbf{K},||$.$) is called a valued field.$

In any non-Archimedean field we have $|1|=|-1|=1$ and $|n| \leq 1$ for $n \in \mathbf{N}_{0}$. In any field $\mathbf{K}$ the function $|\cdot|: \mathbf{K} \rightarrow \mathbf{R}_{+}$given by

$$
|x|:= \begin{cases}0, & x=0, \\ 1, & x=0,\end{cases}
$$

is a valuation which is called trivial, but the most important examples of non-Archimedean fields are $p$-adic numbers which have gained the interest of physicists for their research in some problems coming from quantum physics, $p$-adic strings and superstrings.

Definition 1.5. Let $X$ be a vector space over a scalar field $\mathbf{K}$ with a nonArchimedean non-trivial valuation $|\cdot|$. A function $\|\cdot\|_{*}: X \rightarrow \mathbf{R}$ is a non-Archimedean norm (valuation) if it satisfies the following conditions:

1. $\|x\|_{*}=0$ if and only if $x=0$,

2. $\|r x\|_{*}=|r|\|x\|_{*}(r \in \mathbf{K}, x \in X)$,

3. The strong triangle inequality (ultrametric); namely : $\|x+y\|_{*} \leq \max \left\{\|x\|_{*},\|y\|_{*}\right\} \quad x, y \in X$. 
Then $\left(X,\|\cdot\|_{*}\right)$ is called a non-Archimedean normed space or an ultrametric normed space.

Definition 1.6. Let $\left\{x_{n}\right\}$ be a sequence in a non-Archimedean normed space $X$.

1. A sequence $\left\{x_{n}\right\}_{n=1}^{\infty}$ in a non-Archimedean space is a Cauchy sequence if the sequence $\left\{x_{n+1}-x_{n}\right\}_{n=1}^{\infty}$ converges to zero;

2. The sequence $\left\{x_{n}\right\}$ is said to be convergent if, there exists $x \in X$ such that, for any $\varepsilon>0$, there is a positive integer $N$ such that $\left\|x_{n}-x\right\|_{*} \leq \varepsilon$, for all $n \geq N$. Then the point $x \in X$ is called the limit of the sequence $\left\{x_{n}\right\}$, which is denoted by $\lim _{n \rightarrow \infty} x_{n}=x$;

3. If every Cauchy sequence in $X$ converges, then the non-Archimedean normed space $X$ is called a non-Archimedean Banach space or an non-Archimedean Banach space.

Definition 1.7. (Linear space)[3] Let $\mathcal{K}$ be an arbitrary field (such as the field of real numbers or of complex numbers, for concrete examples). $A$ nonempty set $V$ of elements $x, y, z, \ldots$ of an arbitrary nature, together with an operation, called vector addition, or simply addition, associating with any two elements $x, y \in V$ an element $z \in V$, called the sum of $x$ and $y$, and denoted by $z=x+y$, as well as an operation associating with any $x \in V$ and $\mathrm{a} \in \mathcal{K}$ an element $w \in V$, called the product or scalar product of $a$ and $x$, and denoted by $w=a \cdot x$, is called a linear space if

1. For all $x, y, z \in V$, the operation of vector addition satisfies:

- Associativity, i.e., $(x+y)+z=x+(y+z)$.

- Commutativity, i.e., $x+y=y+x$.

- Existence of a neutral element, i.e., there exists an element $0_{V} \in$ $V$, for which $x+0_{V}=x=0_{V}+x$.

- Existence of additive inverses, i.e., there exists an element $x^{\prime} \in V$ such that $x+x^{\prime}=0_{V}=x^{\prime}+x$.

2. For all $x \in V$ and $\alpha, \beta \in \mathcal{K}$, the scalar product operation satisfies:

- Associativity, i.e., $\alpha \cdot(\beta \cdot x)=(\alpha \beta) \cdot x$.

- Neutrality of $1_{V} \in \mathcal{K}$, i.e., $11_{V} \cdot x=x$. 
3. For all $x, y \in V$ and $\alpha, \beta \in \mathcal{K}$, the scalar product and vector addition operations are compatible in the sense that

- Scalar product distributes over vector addition $\alpha \cdot(x+y)=$ $\alpha \cdot x+\alpha \cdot y$.

- Scalar product distributes over scalar addition $(\alpha+\beta) \cdot x=$ $\alpha \cdot x+\beta \cdot x$.

A linear space is also called a vector space, its elements are called vectors, and the elements of the field $\mathcal{K}$ are called scalars.

Throughout this paper, we will denote the set of natural numbers by $\mathbf{N}, \mathbf{N}_{0}:=\mathbf{N} \cup\{0\}$, the set of real numbers by $\mathbf{R}, \mathbf{R}_{+}=[0, \infty)$ the set of non negative real numbers and $\mathbf{R}_{0}=\mathbf{R} \backslash\{0\}$. By $\mathbf{N}_{m 0}, m_{0} \in \mathbf{N}$, we will denote the set of all natural numbers greater than or equal to $m_{0}$.

Let $X$ be a linear space and let $p \in \mathbf{N}_{3}$ be an odd natural number. We introduce the following functional equation

(1.5) $f\left(\sqrt[p]{x^{p}+y^{p}}\right)+f\left(\sqrt[p]{x^{p}-y^{p}}\right)=2 f(x)+f(y)+f(-y) \quad x, y \in \mathbf{R}$,

where $f: \mathbf{R} \rightarrow X$ which is called $p$-radical functional equation related to Drygas equation (1.4).

The main purpose of this paper is to achieve the general solution of the functional equation (1.5) and establish some hyperstability results for the considered equation in non-Archimedean Banach space. We also provide some corollaries and outcomes concerning the hyperstability results for the inhomogeneous of $p$-radical functional equation.

Before proceeding to the main results, we state Theorem 1.8 which is useful for our purpose. To present it, we introduce the following three hypotheses:

(H1) $X$ is a nonempty set, $Y$ is an non-Archimedean Banach space over a non-Archimedean field, $f_{1}, \ldots, f_{k}: X \longrightarrow X$ and $L_{1}, \ldots, L_{k}: X \longrightarrow$ $\mathbf{R}_{+}$are given.

(H2) $\mathcal{T}: Y^{X} \longrightarrow Y^{X}$ is an operator satisfying the inequality

$$
\|\mathcal{T} \xi(x)-\mathcal{T} \mu(x)\|_{*} \leq \max _{1 \leq i \leq k}\left\{L_{i}(x)\left\|\xi\left(f_{i}(x)\right)-\mu\left(f_{i}(x)\right)\right\|_{*}\right\},
$$




$$
\xi, \mu \in Y^{X}, \quad x \in X
$$

(H3) $\Lambda: \mathbf{R}_{+}^{X} \longrightarrow \mathbf{R}_{+}^{X}$ is a linear operator defined by

$$
\Lambda \delta(x):=\max _{1 \leq i \leq k}\left\{L_{i}(x) \delta\left(f_{i}(x)\right)\right\}, \quad \delta \in \mathbf{R}_{+}^{X}, \quad x \in X .
$$

Thanks to a result due to J. Brzdęk and K. Ciepliński [13, Remark $2]$, we state an analogue of the fixed point theorem [13, Theorem 1] in non-Archimedean Banach space. We use it to assert the existence of a uniquefixed point of operator $\mathcal{T}: Y^{X} \longrightarrow Y^{X}$.

Theorem 1.8. Let hypotheses $(\mathbf{H 1})-(\mathbf{H} 3)$ be valid and functions $\varepsilon: X \longrightarrow$ $\mathbf{R}_{+}$and $\varphi: X \longrightarrow Y$ fulfil the following two conditions

$$
\begin{gathered}
\|\mathcal{T} \varphi(x)-\varphi(x)\|_{*} \leq \varepsilon(x), \quad x \in X, \\
\lim _{n \rightarrow \infty} \Lambda^{n} \varepsilon(x)=0, \quad x \in X .
\end{gathered}
$$

Then there exists a unique fixed point $\psi \in Y^{X}$ of $\mathcal{T}$ with

$$
\|\varphi(x)-\psi(x)\|_{*} \leq \sup _{n \in \mathbf{N}_{0}} \Lambda^{n} \varepsilon(x), \quad x \in X .
$$

Moreover

$$
\psi(x):=\lim _{n \rightarrow \infty} \mathcal{T}^{n} \varphi(x), \quad x \in X
$$

\section{Main results}

The next theorem can be derived from ([18], Corollary 2.3 and Proposition 2.4(a)). However, for the convenience of readers we present it with a directproof.

Theorem 2.1. Let $Y$ be a linear space and $p \in \mathbf{N}_{3}$ be an odd natural number. A function $f: \mathbf{R} \rightarrow Y$ satisfies the functional equation (1.5) if and only if

$$
f(x)=D\left(x^{p}\right), \quad x \in \mathbf{R}
$$

where $D: \mathbf{R} \rightarrow Y$ is a Drygas function. 
Proof. First, if $f: \mathbf{R} \rightarrow Y$ satisfies (2.1) for all $x \in \mathbf{R}$, then, for each $x, y \in \mathbf{R}$, we get that

$$
\begin{aligned}
f\left(\sqrt[p]{x^{p}+y^{p}}\right)+f\left(\sqrt[p]{x^{p}-y^{p}}\right) & =D\left(x^{p}+y^{p}\right)+D\left(x^{p}-y^{p}\right) \\
& =2 D\left(x^{p}\right)+D\left(y^{p}\right)+D\left((-y)^{p}\right) \quad \text { which means } \\
& =2 f(x)+f(y)+f(-y)
\end{aligned}
$$

that $f$ is a solution of equation (1.5). Also, if $f: \mathbf{R} \rightarrow Y$ is a solution of (1.5), then we can write $D(x)=f(\sqrt[p]{x})$ for all $x \in \mathbf{R}$ to find that

$$
\begin{aligned}
D(x+y)+D(x-y) & =f(\sqrt[p]{x+y})+f(\sqrt[p]{x-y}) \\
& =2 f(\sqrt[p]{x})+f(\sqrt[p]{y})+f(\sqrt[p]{-y}) \\
& =2 D(x)+D(y)+D(-y),
\end{aligned}
$$

for all $x, y \in \mathbf{R}$.

Next, we examine the hyperstability of the equation (1.5) in non-

Archimedean Banach space by using, as a basic tool, the fixed point Theorem 1.8.

Theorem 2.2. Let $p$ be an odd natural number, $\left(X,\|\cdot\|_{*}\right)$ be a nonArchimedean Banach space and let $h_{1}, h_{2}: \mathbf{R}_{0} \rightarrow \mathbf{R}_{+}$be two functions such that

$$
\begin{gathered}
\mathcal{U}: \\
=\left\{n \in \mathbf{N}: \alpha_{n}=\max \left\{\lambda_{1}(n+1) \lambda_{2}(n+1), \lambda_{1}(2 n+1) \lambda_{2}(2 n+1)\right.\right. \\
\left.\left.\lambda_{1}(n) \lambda_{2}(n) \lambda_{1}(-n) \lambda_{2}(-n)\right\}<1\right\} \neq \phi,
\end{gathered}
$$

where

$$
\lambda_{i}(m):=\inf \left\{t \in \mathbf{R}_{+}: h_{i}(m x) \leq t h_{i}(x), \quad x \in \mathbf{R}_{0}\right\},
$$

for all $m \in \mathbf{N}$, where $i=1,2$ such that

$$
\lim _{m \rightarrow \infty} \lambda_{1}(m+1) \lambda_{2}(m)=0 .
$$

Assume that $f: \mathbf{R} \rightarrow X$ satisfies the inequality

$$
\left\|f\left(\sqrt[p]{x^{p}+y^{p}}\right)+f\left(\sqrt[p]{x^{p}-y^{p}}\right)-2 f(x)-f(y)-f(-y)\right\|_{*} \leq h_{1}\left(x^{p}\right) h_{2}\left(y^{p}\right),
$$

for all $x, y \in \mathbf{R}_{0}$ such that $x \neq y$ and $x \neq-y$. Then $f$ is a solution of the equation (1.5) on $\mathbf{R}_{0}$.

Proof. $\quad$ Replacing $x$ by $\sqrt[p]{m+1} x$ and $y$ by $\sqrt[p]{m} x$ in the inequality (2.3), we get 


$$
\begin{gathered}
\|2 f(\sqrt[p]{m+1} x)-f(\sqrt[p]{2 m+1} x)+f(\sqrt[p]{m} x)+f(\sqrt[p]{-m} x)-f(x)\|_{*} \\
\leq h_{1}\left((m+1) x^{p}\right) h_{2}\left((m) x^{p}\right),
\end{gathered}
$$

for all $x \in \mathbf{R}_{0}$. For each $m \in \mathbf{N}$, we define the operator $\mathcal{T}_{m}: X^{\mathbf{R}_{0}} \rightarrow X^{\mathbf{R}_{0}}$ by

$$
\mathrm{T}_{m} \xi(x):=2 \xi(\sqrt[p]{m+1} x)-\xi(\sqrt[p]{2 m+1} x)+\xi(\sqrt[p]{m} x)+\xi(\sqrt[p]{-m} x),
$$

for all $\xi \in X^{\mathbf{R}_{0}}, x \in \mathbf{R}_{0}$ and the function $\varepsilon_{m}: \mathbf{R}_{0} \rightarrow \mathbf{R}_{+}$by

$$
\varepsilon_{m}(x):=h_{1}\left((m+1) x^{p}\right) h_{2}\left((m) x^{p}\right), \quad m \in \mathbf{N}, \quad x \in \mathbf{R}_{0} .
$$

We observe that

$$
\varepsilon_{m}(x) \leq \lambda_{1}(m+1) \lambda_{2}(m) h_{1}\left(x^{p}\right) h_{2}\left(x^{p}\right),
$$

for all $x \in \mathbf{R}_{0}$ and all $m \in \mathcal{U}$. Then the inequality (2.4) become as

$$
\left\|\mathcal{T}_{m} f(x)-f(x)\right\|_{*} \leq \varepsilon_{m}(x), \quad x \in \mathbf{R}_{0}
$$

Furthermore, the operator $\Lambda_{m}: \mathbf{R}_{+}^{\mathbf{R}_{0}} \rightarrow \mathbf{R}_{+}^{\mathbf{R}_{0}}$ defined by

$$
\Lambda_{m} \delta(x):=\max _{1 \leq i \leq 4}\left\{L_{i}(x) \delta\left(f_{i}(x)\right\}\right.
$$

for all $x \in \mathbf{R}_{0}$ and all $\delta \in \mathbf{R}_{+}^{\mathbf{R}_{0}}$ where $f_{1}(x)=\sqrt[p]{m+1}$

$f_{2}(x)=\sqrt[p]{2 m+1} x, f_{3}(x)=\sqrt[p]{m} x, f_{4}(x)=\sqrt[p]{-m} x$, and $L_{1}(x)=L_{2}(x)=L_{3}(x)=L_{4}(x)=1$.

Moreover, for every $x \in \mathbf{R}_{0}, \xi, \mu \in X^{\mathbf{R}_{0}}$, we obtain 


$$
\begin{aligned}
& \left\|\mathcal{T}_{m} \xi(x)-\mathcal{T}_{m} \mu(x)\right\|_{*} \\
= & \| 2(\xi(\sqrt[p]{m+1} x)-\mu(\sqrt[p]{m+1} x))-(\xi(\sqrt[p]{2 m+1} x) \\
& -\mu(\sqrt[p]{2 m+1} x)+(\xi(\sqrt[p]{m} x)-\mu(\sqrt[p]{m} x))+(\xi(\sqrt[p]{-m} x)-\mu(\sqrt[p]{-m} x)) \|_{*} \\
\leq & \max \left\{2\|\xi(\sqrt[p]{2 m+1} x)-\mu(\sqrt[p]{2 m+1} x)\|_{*},\right. \\
& \|\xi(\sqrt[p]{m+1} x)-\mu(\sqrt[p]{m+1} x)\|_{*}\|\xi(\sqrt[p]{m} x)-\mu(\sqrt[p]{m} x)\|_{*} \\
& \left.\|\xi(\sqrt[p]{-m} x)-\mu(\sqrt[p]{-m} x)\|_{*}\right\} \\
\leq & \max \left\{\|\xi(\sqrt[p]{2 m+1} x)-\mu(\sqrt[p]{2 m+1} x)\|_{*}, \| \xi(\sqrt[p]{m+1} x)\right. \\
& \left.-\mu(\sqrt[p]{m+1} x)\|,\| \xi(\sqrt[p]{m} x)-\mu(\sqrt[p]{m} x)\left\|_{*},\right\| \xi(\sqrt[p]{-m} x)-\mu(\sqrt[p]{-m} x) \|_{*}\right\} \\
= & \max _{1 \leq i \leq 4}\left\{L_{i}(x)\left\|\xi\left(f_{i}(x)\right)-\mu\left(f_{i}(x)\right)\right\|_{*}\right\},
\end{aligned}
$$

which means that $(\mathbf{H} 2)$ is valid. Now we will show, by induction on $n \in \mathbf{N}_{0}$,

that

$$
\Lambda^{n} \varepsilon_{m}(x) \leq \lambda_{1}(m+1) \lambda_{2}(m) \alpha_{m}^{n} h_{1}\left(x^{p}\right) h_{2}\left(x^{p}\right) .
$$

for all $x \in \mathbf{R}_{0}$ and all $m \in \mathcal{U}$ where

$$
\begin{gathered}
\alpha_{m}=\max \left\{\lambda_{1}(m+1) \lambda_{2}(m+1), \lambda_{1}(2 m+1) \lambda_{2}(2 m+1), \lambda_{1}(m) \lambda_{2}(m),\right. \\
\left.\lambda_{1}(-m) \lambda_{2}(-m)\right\} .
\end{gathered}
$$

For $n=0$, the inequality (2.6) is exactly (2.5). Next we will assume that (2.6) holds for $n=k$, where $k \in \mathbf{N}$. Then

$$
\begin{aligned}
& \Lambda_{m}^{k+1} \varepsilon_{m}(x)=\Lambda_{m}\left(\Lambda_{m}^{k} \varepsilon_{m}(x)\right) \\
= & \max \left\{\Lambda_{m}^{k} \varepsilon_{m}(\sqrt[p]{m+1} x), \Lambda_{m}^{k} \varepsilon_{m}(\sqrt[p]{2 m+1} x), \Lambda_{m}^{k} \varepsilon_{m}(\sqrt[p]{m} x),\right. \\
& \left.\Lambda_{m}^{k} \varepsilon_{m}(\sqrt[p]{-m} x)\right\} \\
\leq & \lambda_{1}(m+1) \lambda_{2}(m) \alpha_{m}^{k} \max \left\{h_{1}\left((m+1) x^{p}\right) h_{2}\left((m+1) x^{p}\right),\right. \\
& h_{1}\left((2 m+1) x^{p}\right) h_{2}\left((2 m+1) x^{p}\right), h_{1}\left((m) x^{p}\right) h_{2}\left((m) x^{p}\right), \\
& \left.h_{1}\left((-m) x^{p}\right) h_{2}\left((-m) x^{p}\right)\right\} \\
\leq & \lambda_{1}(m+1) \lambda_{2}(m) \alpha_{m}^{k} \max \left\{\lambda_{1}(m+1) \lambda_{2}(m+1), \lambda_{1}(2 m+1) \lambda_{2}(2 m+1)\right. \\
& \left.\lambda_{1}(m) \lambda_{2}(m), \lambda_{1}(-m) \lambda_{2}(-m)\right\} h_{1}\left(x^{p}\right) h_{2}\left(x^{p}\right) \\
= & \lambda_{1}(m+1) \lambda_{2}(m) \alpha_{m}^{k+1} h_{1}\left(x^{p}\right) h_{2}\left(x^{p}\right),
\end{aligned}
$$


for all $x \in \mathbf{R}_{0}$ and all $m \in \mathcal{U}$. It shows that (2.6) holds for $n=k+1$. We conclude that the inequality (2.6) holds for all $n \in \mathbf{N}_{0}$.

Since $\alpha_{m}<1$ for all $m \in \mathcal{U}$, we get

$$
\lim _{n \rightarrow \infty} \Lambda^{n} \varepsilon_{m}(x)=0
$$

for all $x \in \mathbf{R}_{0}$. According to Theorem 1.8, there exists, for each $m \in \mathcal{U}$, a fixed point $\mathcal{F}_{m}: \mathbf{R}_{0} \rightarrow X$ of the operator $\mathcal{T}_{m}$ such that

$$
\begin{aligned}
\left\|f(x)-\mathcal{F}_{m}(x)\right\|_{*} & \leq \sup _{n \in \mathbf{N}}\left\{\Lambda_{m}^{n} \varepsilon_{m}(x)\right\} \\
& \leq \sup _{n \in \mathbf{N}}\left\{\lambda_{1}(m+1) \lambda_{2}(m) \alpha_{m}^{n} h_{1}\left(x^{p}\right) h_{2}\left(x^{p}\right)\right\}, \quad x \in \mathbf{R}_{0} .
\end{aligned}
$$

Moreover,

$$
\mathcal{F}_{m}(x)=\lim _{n \rightarrow \infty}\left(\mathcal{T}_{m}^{n} f\right)(x), \quad x \in \mathbf{R}_{0}
$$

Next, we should prove the following inequality

$$
\left\|\mathcal{T}_{m}^{n} f\left(\sqrt[p]{x^{p}+y^{p}}\right)+\mathcal{T}_{m}^{n} f\left(\sqrt[p]{x^{p}-y^{p}}\right)-2 \mathcal{T}_{m}^{n} f(x)-\mathcal{T}_{m}^{n} f(y)-\mathcal{T}_{m}^{n} f(-y)\right\|_{*}
$$

$$
\leq \alpha_{m}^{n} h_{1}\left(x^{p}\right) h_{2}\left(y^{p}\right),
$$

for all $m \in \mathcal{U}$, all $x, y \in \mathbf{R}_{0}$ such that $x \neq y, x \neq-y$ and all $n \in \mathbf{N}$.

We proceed by induction that the case $n=0$ gives us (2.3). Assume that (2.8) holds for $n=k$ where $k \in \mathbf{N}$. Then for each $m \in \mathcal{U}$ and every $x, y \in \mathbf{R}_{0}$ such that $x \neq y$ and $x \neq-y$, we have 


$$
\begin{aligned}
& \left\|\mathcal{T}_{m}^{k+1} f\left(\sqrt[p]{x^{p}+y^{p}}\right)+\mathcal{T}_{m}^{k+1} f\left(\sqrt[p]{x^{p}-y^{p}}\right)-2 \mathcal{T}_{m}^{k+1} f(x)-\mathcal{T}_{m}^{k+1} f(y)-\mathcal{T}_{m}^{k+1} f(-y)\right\|_{*} \\
& =\| 2 \mathcal{T}_{m}^{k} f\left(\sqrt[p]{m+1} \sqrt[p]{x^{p}+y^{p}}\right)-\mathcal{T}_{m}^{k} f\left(\sqrt[p]{2 m+1} \sqrt[p]{x^{p}+y^{p}}\right)+\mathcal{T}_{m}^{k} f\left(\sqrt[p]{m} \sqrt[p]{x^{p}+y^{p}}\right) \\
& +\mathcal{T}_{m}^{k} f\left(\sqrt[p]{-m} \sqrt[p]{x^{p}+y^{p}}\right)+2 \mathcal{T}_{m}^{k} f\left(\sqrt[p]{m+1} \sqrt[p]{x^{p}-y^{p}}\right)-\mathcal{T}_{m}^{k} f\left(\sqrt[p]{2 m+1} \sqrt[p]{x^{p}-y^{p}}\right) \\
& +\mathcal{T}_{m}^{k} f\left(\sqrt[p]{m} \sqrt[p]{x^{p}-y^{p}}\right)+\mathcal{T}_{m}^{k} f\left(\sqrt[p]{-m} \sqrt[p]{x^{p}-y^{p}}\right)-4 \mathcal{T}_{m}^{k} f(\sqrt[p]{m+1} x) \\
& +2 \mathcal{T}_{m}^{k} f(\sqrt[p]{2 m+1} x)-2 \mathcal{T}_{m}^{k} f(\sqrt[p]{m} x)-2 \mathcal{T}_{m}^{k} f(\sqrt[p]{-m} x)-2 \mathcal{T}_{m}^{k} f(\sqrt[p]{m+1} y) \\
& +\mathcal{T}_{m}^{k} f(\sqrt[p]{2 m+1} y)-\mathcal{T}_{m}^{k} f(\sqrt[p]{m} y)-\mathcal{T}_{m}^{k} f(\sqrt[p]{-m} y)-2 \mathcal{T}_{m}^{k} f(\sqrt[p]{m+1}(-y)) \\
& +\mathcal{T}_{m}^{k} f(\sqrt[p]{2 m+1}(-y))-\mathcal{T}_{m}^{k} f(\sqrt[p]{m}(-y))-\mathcal{T}_{m}^{k} f(\sqrt[p]{-m}(-y)) \|_{*} \\
& \leq \max \left\{\| \mathcal{T}_{m}^{k} f\left(\sqrt[p]{m+1} \sqrt[p]{x^{p}+y^{p}}\right)+\mathcal{T}_{m}^{k} f\left(\sqrt[p]{m+1} \sqrt[p]{x^{p}-y^{p}}\right)-2 \mathcal{T}_{m}^{k} f(\sqrt[p]{m+1} x)\right. \\
& -\mathcal{T}_{m}^{k} f(\sqrt[p]{m+1} y)-\mathcal{T}_{m}^{k} f(\sqrt[p]{m+1}(-y)) \|_{*}, \\
& \| \mathcal{T}_{m}^{k} f\left(\sqrt[p]{2 m+1} \sqrt[p]{x^{p}+y^{p}}\right)+\mathcal{T}_{m}^{k} f\left(\sqrt[p]{2 m+1} \sqrt[p]{x^{p}-y^{p}}\right)-2 \mathcal{T}_{m}^{k} f(\sqrt[p]{2 m+1} x) \\
& -\mathcal{T}_{m}^{k} f(\sqrt[p]{2 m+1} y)-\mathcal{T}_{m}^{k} f(\sqrt[p]{2 m+1}(-y)) \|_{*}, \\
& \| \mathcal{T}_{m}^{k} f\left(\sqrt[p]{m} \sqrt[p]{x^{p}+y^{p}}\right)+\mathcal{T}_{m}^{k} f\left(\sqrt[p]{m} \sqrt[p]{x^{p}-y^{p}}\right)-2 \mathcal{T}_{m}^{k} f(\sqrt[p]{m} x) \\
& -\mathcal{T}_{m}^{k} f(\sqrt[p]{m} y)-\mathcal{T}_{m}^{k} f(\sqrt[p]{m}(-y)) \|_{*}, \\
& \| \mathcal{T}_{m}^{k} f\left(\sqrt[p]{-m} \sqrt[p]{x^{p}+y^{p}}\right)+\mathcal{T}_{m}^{k} f\left(\sqrt[p]{-m} \sqrt[p]{x^{p}-y^{p}}\right)-2 \mathcal{T}_{m}^{k} f(\sqrt[p]{-m} x) \\
& \left.-\mathcal{T}_{m}^{k} f(\sqrt[p]{-m} y)-\mathcal{T}_{m}^{k} f(\sqrt[p]{-m}(-y)) \|_{*}\right\} \\
& \leq \max \left\{\alpha_{m}^{k} h_{1}\left((m+1) x^{p}\right) h_{2}\left((m+1) y^{p}\right), \alpha_{m}^{k} h_{1}\left((2 m+1) x^{p}\right) h_{2}\left((2 m+1) y^{p}\right)\right. \text {, } \\
& \left.\alpha_{m}^{k} h_{1}\left((m) x^{p}\right) h_{2}\left((m) y^{p}\right), \alpha_{m}^{k} h_{1}\left((-m) x^{p}\right) h_{2}\left((-m) y^{p}\right)\right\} \\
& \leq \alpha_{m}^{k} h_{1}\left(x^{p}\right) h_{2}\left(y^{p}\right) \max \left\{\lambda_{1}(m+1) \lambda_{2}(m+1), \lambda_{1}(2 m+1) \lambda_{2}(2 m+1)\right. \text {, } \\
& \left.\lambda_{1}(m) \lambda_{2}(m), \lambda_{1}(-m) \lambda_{2}(-m)\right\} \\
& =\alpha_{m}^{k+1} h_{1}\left(x^{p}\right) h_{2}\left(y^{p}\right) \text {. }
\end{aligned}
$$

Thus, we have shown that (2.8) holds for $n \in \mathbf{N}_{0}$, and all $m \in \mathcal{U}$. Letting $n \rightarrow \infty$ in (2.8), we obtain

$$
\mathcal{F}_{m}\left(\sqrt[p]{x^{p}+y^{p}}\right)+\mathcal{F}_{m}\left(\sqrt[p]{x^{p}-y^{p}}\right)=2 \mathcal{F}_{m}(x)+\mathcal{F}_{m}(y)+\mathcal{F}_{m}(-y),
$$

for all $x, y \in \mathbf{R}_{0}$ such that $x \neq y, x \neq-y$ and $m \in \mathcal{U}$. This implies that $\mathcal{F}_{m}: \mathbf{R} \rightarrow X$ is a solution of the equation (1.5). 
Therefore, we construct a sequence $\left\{\mathcal{F}_{m}\right\}_{m \in \mathcal{U}}$ of the solutions of equation (1.5) on $\mathbf{R}_{0}$ such that

$$
\begin{aligned}
\left\|\mathcal{F}_{m}(x)-f(x)\right\|_{*} & \leq \sup _{n \in \mathbf{N}} \Lambda_{m}^{n} \varepsilon_{m}(x) \\
& \leq \sup _{n \in \mathbf{N}}\left\{\lambda_{1}(m+1) \lambda_{2}(m) \alpha_{m}^{n} h_{1}\left(x^{p}\right) h_{2}\left(x^{p}\right)\right\},
\end{aligned}
$$

for all $x \in \mathbf{R}_{0}$ and all $m \in \mathcal{U}$. Letting $n \rightarrow \infty$ in the previous inequality and using (2.2), we deduce that $f$ is a solution of the equation (1.5) on $\mathbf{R}_{0}$ which means that the equation (1.5) is hyperstable on $\mathbf{R}_{0}$.

In a similar way, we can prove the following theorem.

Theorem 2.3. Let $p$ be an odd natural number, $\left(X,\|\cdot\|_{*}\right)$ be a nonArchimedean Banach space and let $h: \mathbf{R}_{0} \rightarrow \mathbf{R}_{+}$be a mapping such that $\mathcal{U}:=\left\{n \in \mathbf{N}: \alpha_{n}=\max \{\lambda(n+1), \lambda(2 n+1), \lambda(n), \lambda(-n)\}<1\right\} \neq \phi$, where

$$
\lambda(n)=\inf \left\{t \in \mathbf{R}_{+}: h(n x) \leq t h(x), \quad x \in \mathbf{R}_{0}\right\},
$$

for all $n \in \mathbf{N}$, such that

$$
\lim _{n \rightarrow \infty}(\lambda(n+1)+\lambda(n))=0 .
$$

Assume that $f: \mathbf{R} \rightarrow X$ satisfies the inequality

$(2.8)\left\|f\left(\sqrt[p]{x^{p}+y^{p}}\right)+f\left(\sqrt[p]{x^{p}-y^{p}}\right)-2 f(x)-f(y)-f(-y)\right\|_{*} \leq h\left(x^{p}\right)+h\left(y^{p}\right)$,

for all $x, y \in \mathbf{R}_{0}$ such that $x \neq y$ and $x \neq-y$. Then $f$ is a solution of the equation (1.5) on $\mathbf{R}_{0}$.

Proof. We will suffice with the basic idea of the proof. Replacing $x$ by $\sqrt[p]{m+1} x$ and $y$ by $\sqrt[p]{m} x$ in the inequality (2.8) where $x \in \mathbf{R}_{0}, m \in \mathcal{U}$, we get

$$
\begin{aligned}
& \|2 f(\sqrt[p]{m+1} x)-f(\sqrt[p]{2 m+1} x)+f(\sqrt[p]{m} x)+f(\sqrt[p]{-m} x)-f(x)\|_{*} \\
& \leq h\left((m+1) x^{p}\right)+h\left((m) x^{p}\right) \\
& \leq(\lambda(m+1)+\lambda(m)) h\left(x^{p}\right),
\end{aligned}
$$

for all $m \in \mathcal{U}$ and all $x \in \mathbf{R}_{0}$. We define operators $\mathcal{T}_{m}: X^{\mathbf{R}_{0}} \rightarrow X^{\mathbf{R}_{0}}$ and $\Lambda_{m}: \mathbf{R}_{+}^{\mathbf{R}_{0}} \rightarrow \mathbf{R}_{+}^{\mathbf{R}_{0}}$ by 


$$
\mathcal{T}_{m} \xi(x):=2 \xi(\sqrt[p]{m+1} x)-\xi(\sqrt[p]{2 m+1} x)+\xi(\sqrt[p]{m} x)+\xi(\sqrt[p]{-m} x)
$$

for all $\xi \in X^{\mathbf{R}_{0}}$ and all $x \in \mathbf{R}_{0}$ and

$\Lambda_{m} \delta(x):=\max \{\delta(\sqrt[p]{m+1} x), \delta(\sqrt[p]{2 m+1} x), \delta(\sqrt[p]{m} x), \delta(\sqrt[p]{-m} x)\}$

Moreover, we write

$\varepsilon_{m}(x)=h\left((m+1) x^{p}\right)+h\left((m) x^{p}\right) \leq(\lambda(m+1)+\lambda(m)) h\left(x^{p}\right), \quad x \in \mathbf{R}_{0}$.

As in Theorem 2.2, we observe that inequality (2.8) takes the following form

$$
\left\|f(x)-\mathcal{T}_{m}(x) \mid\right\|_{*} \leq \varepsilon_{m}(x), \quad x \in \mathbf{R}_{0}, m \in \mathcal{U},
$$

then we complete the proof by similar steps of the proof of Theorem 2.2.

\section{Consequences}

In this section, we get, as particular cases of our main results, the hyperstability results in the sense of Hyers-Ulam-Rassiass. Also, we get the same results for the inhomogeneous general $p$-radical functional equation

$$
f\left(\sqrt[p]{x^{p}+y^{p}}\right)+f\left(\sqrt[p]{x^{p}-y^{p}}\right)=2 f(x)+f(y)+f(-y)+G(x, y) .
$$

Corollary 3.1. Let $p$ be an odd natural number, $\left(X,\|\cdot\|_{*}\right)$ be a nonArchimedean Banach space and let $c, r, s \in \mathbf{R}$ such that $r+s<0$ and $c \geq 0$. Assume that a function $f: \mathbf{R} \rightarrow X$ satisfies the inequality

$\left\|f\left(\sqrt[p]{x^{p}+y^{p}}\right)+f\left(\sqrt[p]{x^{p}-y^{p}}\right)-2 f(x)-f(y)-f(-y)\right\|_{*} \leq c\left|Q_{1}\left(x^{p}\right)\right|^{r}\left|Q_{2}\left(y^{p}\right)\right|^{s}$,

for all $x, y \in \mathbf{R}_{0}$ where $Q_{1}, Q_{2}: \mathbf{R} \rightarrow \mathbf{R}_{+}$are two quadratic mappings. Then $f$ is a solution of the equation (1.5) on $\mathbf{R}_{0}$. 
Proof. The proof follows from Theorem 2.2 by taking $h_{1}, h_{2}: \mathbf{R}_{0} \rightarrow \mathbf{R}_{+}$ as follows:

$$
h_{1}\left(x^{p}\right)=c_{1}\left|Q_{1}\left(x^{p}\right)\right|^{r}
$$

and

$$
h_{2}\left(x^{p}\right)=c_{2}\left|Q_{2}\left(x^{p}\right)\right|^{s}
$$

for all $x, y \in \mathbf{R}_{0}$ where $c_{1}, c_{2} \in \mathbf{R}_{+}$such that $c_{1} c_{2}=c \geq 0$. For each $m \in \mathbf{N}$, we define $\lambda_{1}(m)$ as in Theorem 2.2 by

$$
\begin{aligned}
\lambda_{1}(m) & =\inf \left\{t \in \mathbf{R}_{+}: h_{1}\left(m x^{p}\right) \leq t h_{1}\left(x^{p}\right)\right\} \\
& =\inf \left\{t \in \mathbf{R}_{+}: c_{1}\left|Q_{1}\left(m x^{p}\right)\right|^{r} \leq t c_{1}\left|Q_{1}\left(x^{p}\right)\right|^{r}\right\} \\
& =\inf \left\{t \in \mathbf{R}_{+}: m^{2 r}\left|Q_{1}\left(x^{p}\right)\right|^{r} \leq t\left|Q_{1}\left(x^{p}\right)\right|^{r}\right. \\
& =m^{2 r},
\end{aligned}
$$

for all $x \in \mathbf{R}_{0}$. Also, for each $m \in \mathbf{N}$, we have $\lambda_{2}(m)=m^{2 s}$. It is clear that there exists $m_{0} \in \mathbf{N}$ such that, for each $m \geq m_{0}$, we get

$$
\begin{aligned}
\alpha_{m}= & \max \left\{\lambda_{1}(m+1) \lambda_{2}(m+1), \lambda_{1}(2 m+1) \lambda_{2}(2 m+1),\right. \\
& \left.\lambda_{1}(m) \lambda_{2}(m), \lambda_{1}(m) \lambda_{2}(m)\right\}, \\
= & \max \left\{(m+1)^{2(r+s)},(2 m+1)^{2(r+s)}, m^{2(r+s)},(-m)^{2(r+s)}\right\}<1
\end{aligned}
$$

According to Theorem 2.2, there exists a unique function $\mathcal{F}_{m}: \mathbf{R}_{0} \rightarrow X$ satisfies the equation (1.5) such that

$$
\begin{aligned}
\left\|\mathcal{F}_{m}-f(x)\right\|_{*} & \leq c \sup _{n \in \mathbf{N}}\left\{\lambda_{1}(m+1) \lambda_{2}(m) \alpha_{m}^{n}\left|Q_{1}\left(x^{p}\right)\right|^{r}\left|Q_{2}\left(x^{p}\right)\right|^{s}\right\} \\
& =c(m+1)^{2 r} m^{4 s}\left|Q_{1}\left(x^{p}\right)\right|^{r}\left|Q_{2}\left(x^{p}\right)\right|^{s} \sup _{n \in \mathbf{N}}\left\{\alpha_{m}^{n}\right\},
\end{aligned}
$$

for all $x \in \mathbf{R}_{0}$. On the other hand, Since $r+s<0$, one of $r, s$ must be negative. Assume that $r<0$. Then

$$
\lim _{m \rightarrow \infty} \lambda_{1}(m+1) \lambda_{2}(m)=\lim _{m \rightarrow \infty} m^{2(r+s)}=0
$$

We get the desired result.

Corollary 3.2. Let $p$ be an odd natural number, $\left(X,\|\cdot\|_{*}\right)$ be a nonArchimedean Banach space and let $c, r \in \mathbf{R}$ such that $c \geq 0$ and $r<0$. Assume that a function $f: \mathbf{R} \rightarrow X$ satisfies the inequality

$\left\|f\left(\sqrt[p]{x^{p}+y^{p}}\right)+f\left(\sqrt[p]{x^{p}-y^{p}}\right)-2 f(x)-f(y)-f(-y)\right\|_{*} \leq c\left(\left|Q\left(x^{p}\right)\right|^{r}+\left|Q\left(y^{p}\right)\right|^{r}\right)$, 
for all $x, y \in \mathbf{R}_{0}$ where $Q: \mathbf{R} \rightarrow \mathbf{R}_{+}$is a quadratic mapping. Then $f$ is a solution of the equation (1.5) on $\mathbf{R}_{0}$.

Proof. The proof is similar to the proof of Corollary 3.1 with taking $h: \mathbf{R}_{0} \rightarrow \mathbf{R}_{+}$defined by $h\left(x^{p}\right)=c\left|Q\left(x^{p}\right)\right|^{r}$ for all $x \in \mathbf{R}_{0}$ where $c \geq 0$ and $r<0$.

In the following corollaries, we get the hyperstability results for the inhomogeneous general $p$-radical functional equation related to quadratic mappings.

Corollary 3.3. Let $p$ be an odd natural number, $c, r, s \in \mathbf{R}$ such that $c \geq 0$ and $r+s<0,\left(X,\|\cdot\|_{*}\right)$ be a non-Archimedean Banach space, $G: \mathbf{R} \times \mathbf{R} \rightarrow X$ be a function such that $G(0,0)=0$ and let $f: \mathbf{R} \rightarrow X$ be a function such that $f(0)=0$. Assume that $f$ and $G$ satisfy the inequality

$$
\left\|f\left(\sqrt[p]{x^{p}+y^{p}}\right)+f\left(\sqrt[p]{x^{p}-y^{p}}\right)-2 f(x)-6 f(y)-f(-y)-G(x, y)\right\|_{*}
$$

$$
\leq c\left|Q_{1}\left(x^{p}\right)\right|^{r}\left|Q_{2}\left(y^{p}\right)\right|^{s},
$$

for all $x, y \in \mathbf{R}_{0}$, where $Q_{1}, Q_{2}: \mathbf{R} \rightarrow \mathbf{R}_{+}$are two quadratic mappings. If the functional equation

$$
f\left(\sqrt[p]{x^{p}+y^{p}}\right)+f\left(\sqrt[p]{x^{p}-y^{p}}\right)-2 f(x)-f(y)-f(-y)-G(x, y)=0
$$

has a solution $f_{0}: \mathbf{R} \rightarrow X$ on $\mathbf{R}_{0}$, then $f$ is a solution of the equation (3.6) on $\mathbf{R}_{0}$.

Proof. Let $\psi: \mathbf{R} \rightarrow X$ be a function defined by $\psi(x):=f(x)-f_{0}(x)$ for all $x \in \mathbf{R}$. Then we get that 


$$
\begin{aligned}
& \left\|\psi\left(\sqrt[p]{x^{p}+y^{p}}\right)+\psi\left(\sqrt[p]{x^{p}-y^{p}}\right)-2 \psi(x)-\psi(y)-\psi(-y)\right\|_{*} \\
& =\| f\left(\sqrt[p]{x^{p}+y^{p}}\right)+f\left(\sqrt[p]{x^{p}-y^{p}}\right)-2 f(x)-f(y)-f(-y) \\
& \quad-G(x, y)-f_{0}\left(\sqrt[p]{x^{p}+y^{p}}\right)-f_{0}\left(\sqrt[p]{x^{p}-y^{p}}\right) \\
& +2 f_{0}(x)+f_{0}(y)+f_{0}(-y)+G(x, y) \|_{*} \\
& \leq \max \left\{\left\|f\left(\sqrt[p]{x^{p}+y^{p}}\right)+f\left(\sqrt[p]{x^{p}-y^{p}}\right)-2 f(x)-f(y)-f(-y)-G(x, y)\right\|_{*},\right. \\
& \left.\left\|f_{0}\left(\sqrt[p]{x^{p}+y^{p}}\right)+f_{0}\left(\sqrt[p]{x^{p}-y^{p}}\right)-2 f_{0}(x)-f_{0}(y)-f_{0}(-y)-G(x, y)\right\|_{*}\right\}^{\prime} \\
& \leq\left\|f\left(\sqrt[p]{x^{p}+y^{p}}\right)+f\left(\sqrt[p]{x^{p}-y^{p}}\right)-2 f(x)-f(y)-f(-y)-G(x, y)\right\|_{*} \\
& \leq c\left|Q_{1}\left(x^{p}\right)\right|^{r}\left|Q_{2}\left(y^{p}\right)\right|^{s},
\end{aligned}
$$

for all $x, y \in \mathbf{R}_{0}$. By using Corollary 3.1, we deduce that $\psi$ is a solution of equation (1.5). Moreover, for all $x, y \in \mathbf{R}_{0}$, we have

$$
\begin{aligned}
& f\left(\sqrt[p]{x^{p}+y^{p}}\right)+f\left(\sqrt[p]{x^{p}-y^{p}}\right)-2 f(x)-f(y)-f(-y)-G(x, y) \\
& =\psi\left(\sqrt[p]{x^{p}+y^{p}}\right)+\psi\left(\sqrt[p]{x^{p}-y^{p}}\right)-2 \psi(x)-\psi(y)-\psi(-y) \\
& +f_{0}\left(\sqrt[p]{x^{p}+y^{p}}\right)+f_{0}\left(\sqrt[p]{x^{p}-y^{p}}\right)-2 f_{0}(x)-f_{0}(y)-f_{0}(-y)-G(x, y)=0,
\end{aligned}
$$

which means that $f$ is a solution of (3.6) on $\mathbf{R}_{0}$.

With an analogous proof of Corollary 3.3, we can prove the following corollary.

Corollary 3.4. Let $p$ be an odd natural number, $c, r \in \mathbf{R}$ such that $c \geq 0$ and $r<0,\left(X,\|\cdot\|_{*}\right)$ be a non-Archimedean Banach space and let $G$ : $\mathbf{R} \times \mathbf{R} \rightarrow X$ be a function such that $G(0,0)=0$ and $f: \mathbf{R} \rightarrow X$ be a function such that $f(0)=0$. Assume that $f$ and $G$ satisfy the inequality

$$
\begin{aligned}
& \left\|f\left(\sqrt[p]{x^{p}+y^{p}}\right)+f\left(\sqrt[p]{x^{p}-y^{p}}\right)-2 f(x)-f(y)-f(-y)-G(x, y)\right\|_{*} \\
& \leq c\left(\left|Q\left(x^{p}\right)\right|^{r}+\left|Q\left(y^{p}\right)\right|^{r}\right)
\end{aligned}
$$

for all $x, y \in \mathbf{R}_{0}$, where $Q: \mathbf{R} \rightarrow \mathbf{R}_{+}$is a quadratic mapping. If the functional equation

$$
f\left(\sqrt[p]{x^{p}+y^{p}}\right)+f\left(\sqrt[p]{x^{p}-y^{p}}\right)-2 f(x)-f(y)-f(-y)-G(x, y)=0
$$

has a solution $f_{0}: \mathbf{R} \rightarrow X$ on $\mathbf{R}_{0}$, then $f$ is a solution of the equation (3.7) on $\mathbf{R}_{0}$. 


\section{References}

[1] L. Aiemsomboon and W. Sintunavarat, "On a new type of stability of a radical quadratic functional equation using Brzdęk's fixed point theorem", Acta mathematica hungarica, vol. 151, no. 1, pp. 35-46, Nov. 2016, doi: 10.1007/s10474-016-0666-2

[2] L. Aiemsomboon and W. Sintunavarat, "On generalized hyperstability of a general linear equation", Acta mathematica hungarica, vol. 149, no. 2, pp. 413-422, May 2016, doi: 10.1007/s10474-016-0621-2

[3] C. Alabiso and I. Weiss, A primer on Hilbert space theory: linear spaces, topological spaces, metric spaces, normed spaces, and topological groups. Cham: Springer, 2015, doi: 10.1007/978-3-319-03713-4.

[4] Z. Alizadeh and A. G. Ghazanfari, "On the stability of a radical cubic functional equation in quasi- $\beta$-spaces", Journal of fixed point theory and applications, vol. 18, no. 4, pp. 843-853, Aug. 2016, doi: $10.1007 /$ s 11784-016-0317-9

[5] M. Almahalebi, A. Charifi and S. Kabbaj, "Hyperstability of a Cauchy functional equation", Journal of nonlinear analysis and optimization: theory applications, vol. 6, no. 2, pp. 127-137, 2015. [On line]. Available: https:// bit.ly/3ny4yMN

[6] M. Almahalebi, "On the hyperstability of $\sigma$-Drygas functional equation on semigroups", Aequationes mathematicae, vol. 90, no. 4, pp. 849-857, Aug. 2016, doi: 10.1007/s00010-016-0422-2

[7] M. Almahalebi and C. Park, "On the hyperstability of a functional equation in commutative groups", Journal of computational analysis applications, vol. 20, no. 1, pp. 826-833, 2016.

[8] T. Aoki, "On the stability of the linear transformation in Banach spaces", Journal of the Mathematical Society of Japan, vol. 2, no. 1-2, pp. 64-66, Sep. 1950, doi: 10.2969/ jms j/ 00210064

[9] D. G. Bourgin, "Classes of transformations and bordering transformations", Bulletin of the American mathematical society, vol. 57, no. 4, pp. 223-237, 1951, doi: 10.1090/S0002-9904-1951-09511-7

[10] N. Brillouët-Belluot, J. Brzdęk, and K. Ciepliński, "On some recent developments in Ulams type stability", Abstract and applied analysis, vol. 2012, pp. Art ID. 716936, Dec. 2012, doi: 10.1155/2012/716936 
[11] J. Brzdęk, "A note on stability of additive mappings", in Stability of mappings of Hyers-Ulam type, T. M. Rassias and J. Tabor, Eds. Palm Harbor, FL: Hadronic Press, 1994, pp. 19-22.

[12] J. Brzdęk, J. Chudziak, and Z. Páles, “A fixed point approach to stability of functional equations", Nonlinear analysis: theory, methods \& applications, vol. 74, no. 17, pp. 6728-6732, Dec. 2011, doi: $10.1016 /$ j.na.2011.06.052

[13] J . Brzdęk and K. Ciepliński, "A fixed point approach to the stability of functional equations in non-Archimedean metric spaces", Nonlinear analysis: theory, methods \& applications, vol. 74, no. 18, pp. 6861-6867, Dec. 2011, doi: 10.1016/ j.na.2011.06.050

[14] J. Brzdęk, "Stability of additivity and fixed point methods", Fixed point theory and applications, vol. 2013, no. 1, Art ID. 285, Nov. 2013, doi: 10.1186/ 1687-1812-2013-285

[15] J. Brzdęk "Hyperstability of the Cauchy equation on restricted domains," Acta mathematica hungarica, vol. 141, no. 1-2, pp. 58-67, Feb. 2013, doi: 10.1007/s10474-013-0302-3

[16] J. Brzdęk, L. Cădariu, and K. Ciepliński, "Fixed point theory and the Ulam stability", Journal of function spaces, vol. 2014, Art ID. 829419, 2014, doi: 10.1155/2014/829419

[17] J. Brzdęk, W. Fechner, M. S. Moslehian, and J. Sikorska, "Recent developments of the conditional stability of the homomorphism equation", Banach journal of mathematical analysis, vol. 9, no. 3, pp. 278-326, 2015, doi:10.15352/bjma/ 09-3-20

[18] J. Brzdęk, "Remarks on solutions to the functional equations of the radical type", Advances in the theory of nonlinear analysis and its application, vol. 1, no. 2, pp. 125-135, Dec. 2017, doi: $10.31197 /$ atnaa. 379095

[19] H. Drygas, "Quasi-inner products and their applications", in Advances in multivariate statistical analysis, A. K. Gupta, Ed. Dordrecht: Springer, 1987,pp. 13-30, doi: 10.1007/978-94-017-0653-7_2

[20] B. R. Ebanks, P. L. Kannappan, and P. K. Sahoo, "A common generalization of functional equations characterizing normed and quasi-inner-product spaces", Canada mathematical bulletin, vol. 35, no. 3, pp. 321-327, Sep. 1992, doi: 10.4153/CMB-1992-044-6

[21] M. E. Gordji, H. Khodaei, A. Ebadian, and G. H. Kim, "Nearly radical quadratic functional equations in $p$-2-normed spaces", Abstract and applied analysis, vol. 2012 Art ID. 896032 , 2012, doi: $10.1155 / 2012 / 896032$ 
[22] M. E. Gordji and M. Parviz, "On the Hyers Ulam stability of the functional equation", Nonlinear functions analysis applications, vol. 14, no. 3, pp. 413-420, 2009. [On line]. Available: https:// bit.ly/3pVAIDN

[23] P. Găvruta, "A generalization of the Hyers-Ulam-Rassias stability of approximately additive mappings", Journal mathematics analysis applications, vol. 184, no. 3, pp. 431-436, Jun. 1994, doi: 10.1006/ jmaa.1994.1211

[24] D. H. Hyers, "On the stability of the linear functional equation", Proceedings of the National Academy of Sciences of the United States of America, vol. 27, no. 4, pp. 222-224, 1941, doi: 10.1073/ pnas.27.4.222

[25] S.-M. Jung, and P. K. Sahoo, "Stability of a functional equation of Drygas", Aequationes mathematicae, vol.64, no.3,pp.263-273, Dec.2002, doi : 10.1007/ PL00012407

[26] H. Khodaei, M. E. Gord ji, S. Kim, and Y. Cho, "Approximation of radical functional equations related to quadratic and quartic mappings", Journal of mathematical analysis and applications, vol. 395, no. 1, pp. 284-297, Nov. 2012, doi: 10.1016/ j.jmaa.2012.04.086

[27] S. Kim, Y. Cho, and M. E. Gordji, "On the generalized Hyers-UlamRassias stability problem of radical functional equations", Journal of inequalities and applications, Art ID. 186, Aug. 2012, doi: 10.1186/ 1029-242X-2012-186

[28] G. Maksa and Z. Pales, "Hyperstability of a class of linear functional equations", Acta Mathematica Academiae Paedagogicae Nyiregyhaziensis, vol. 17, no. 2, pp. 107-112, 2001. [On line]. Available: https:/ / bit.ly/3nzQLFv

[29] M. Piszczek and J. Szczawińska, "Hyperstability of the Drygas functional equation", Journal function spaces and applied, vol. 2013, Art ID 912718, Jul. 2013, doi: 10.1155/2013/912718

[30] University of Cracow and AGH University of Science and Technology "16th International Conference on Functional Equations and Inequalities, Będlewo, Poland, May 17-23, 2015”, Annales Universitatis Paedagogicae Cracoviensis. Studia Mathematica, vol. 14, no. 1, pp. 163-202, Dec. 2015, doi: 10.1515/ aupcsm-2015-0012

[31] Th. M. Rassias, "On the stability of the linear mapping in Banach spaces", Proceedings of the American mathematical society, vol. 72, pp. 297-300, 1978, doi: 10.1090/ S0002-9939-1978-0507327-1

[32] Th. M. Rassias, "Problem 16, $2^{\circ}$. The Twenty-seventh International Symposium on Functional Equations, August 14-24, 1989, Bielsko-BiałKatowice-Kraków, Poland", Aequationes mathematicae, vol. 39, no. 2-3, pp. 292-293, Apr. 1990, doi: 10.1007/ BF01833155 
[33] Th. M. Rassias, "On a modified Hyers-Ulam sequence”, Journal of mathematical analysis and applications, vol. 158, no. 1, pp. 106-113, Jun. 1991, doi: 10.1016/ 0022-247X(91)90270-A

[34] P. K. Sahoo and P. Kannappan, Introduction to functional equations. Boca Raton, FL: CRCPress, 2011.

[35] M. Sirouni and S. Kabbaj, "A fixed point approach to the hyperstability of Drygas functional equation in metric spaces", Journal of mathematical and computational science, vol. 4, no. 4, 2014, 705-715. [On line]. Available: https:// bit.ly/2MXuvJb

[36] W. Smajdor, "On set-valued solutions of a functional equation of Drygas", Aequationes mathematicae, vol. 77, pp. 89-97, Mar. 2009, doi: $10.1007 / \mathrm{s} 00010-008-2935-9$

[37] S. M. Ulam, Problems in modern mathematics. New York, NY: J. Wiley, 1964. 